\title{
Participant and parent experiences in the oral insulin study of the Diabetes Prevention Trial for Type 1 Diabetes
}

\author{
Suzanne Bennett Johnson ${ }^{\mathrm{a}}$, Amy E Baughcum ${ }^{\mathrm{b}}$, Lisa E Rafkin-Mervis ${ }^{\mathrm{c}}$, and Desmond A \\ Schatz ${ }^{d}$ for the DPT-1 Study Group \\ aDepartment of Medical Humanities and Social Sciences, Florida State University College of \\ Medicine, Tallahassee, FL, USA \\ bDepartment of Psychology, Nationwide Children's Hospital, Columbus, OH, USA \\ 'Department of Medicine, Research Institute, University of Miami Medical School, Miami, FL, USA \\ dDepartment of Pediatrics, University of Florida College of Medicine, Gainesville, FL, USA
}

\section{Abstract}

Objective-To assess the experiences of participants and parents of children in the oral insulin study of the Diabetes Prevention Trial -Type 1.

Method-Before trial results were publicized, surveys were completed by 124 participants and 219 parents of children in the oral trial.

Results-Although most of those surveyed were positive about the trial, survey results suggest that participant perspective (adult, child, parent, and gender), study procedures, and beliefs about placebo vs. active drug assignment have important implications for planning future clinical trials. Parents and children reported greater distress, worry, and difficulty making the decision to join the trial compared with adult participants. Mothers and female participants were particularly interested in additional psychosocial support during the study. Random assignment was viewed negatively by both parents and children, and close observation for diabetes onset was viewed as the most favorable aspect of the study. Adherence to study procedures declined over time and behaviors outside the study protocol to prevent/delay diabetes onset were common, particularly among those who believed the participant was taking a placebo. Children and respondents who believed that the participant was taking the active drug expressed confidence in oral insulin's ability to delay or prevent type 1 diabetes.

Conclusions-Although most participants were positive about the trial and many expressed optimism about the intervention's potential for success, future trials need to address negative reactions to random assignment, the unique concerns of children and their parents, declining adherence, and behaviors - external to the trial - designed to delay or prevent diabetes.

\footnotetext{
(C) 2008 The Authors

Corresponding author: Suzanne Bennett Johnson, Department of Medical Humanities and Social Sciences Florida State University College of Medicine 1115 West Call Street Tallahassee, FL 2306-4300 USA. Tel: 850644 3457; fax: 850645 1773; suzanne.johnson@med.fsu.edu.
} 


\section{Keywords}

primary prevention; protocol compliance; randomized controlled trials; research subjects; type 1 diabetes mellitus

The Diabetes Prevention Trial - Type 1 (DPT-1) tested whether insulin could prevent or delay type 1 diabetes onset in relatives of type 1 patients. There were two separate prevention trials. Relatives with $\geq 50 \%$ risk of developing diabetes within $5 \mathrm{yr}$ were offered randomization to a parenteral insulin intervention or a closely monitored control condition. Relatives with a 5-yr risk of 26-50\% were offered randomization to oral insulin therapy or a placebo (1). We have previously described participant and parent experiences in the parenteral insulin trial (2). Presented in this study are the experiences of those in the oral insulin trial. All oral insulin participants were blind to condition (active drug vs. placebo), and all were asked to take one capsule a day before breakfast. All underwent home blood glucose assessments every 3 months or when they suspected hypoglycemia, and all were administered an oral glucose tolerance test (OGTT) every 6 months. At the end of this trial, before the results were known, participants $\geq 10 \mathrm{yr}$ of age and parents of children in the trial were asked to complete a survey about their study experiences. Although oral insulin failed to prevent or delay type 1 diabetes in the DPT-1, there was some suggestion of positive benefit in participants with islet cell autoantibodies and higher titers of insulin autoantibodies ( $\ 80 \mathrm{nU} / \mathrm{mL}$ ) (3). This hypothesis is being tested in a follow-up study as part of Type 1 Diabetes TrialNet. Consequently, participant and parent perceptions and experiences in the DPT-1 oral insulin trial can help inform the TrialNet oral insulin study as well as other prevention trials.

\section{Methods}

\section{Survey development}

A survey (5th grade reading level) was developed to address (i) distress at the beginning of the trial; (ii) decision making around trial participation; (iii) beliefs about group assignment (active drug vs. placebo); (iv) reactions to study procedures; (v) adherence with study demands; (vi) efforts to prevent diabetes; (vii) need for psychological support; and (viii) beliefs about the use of oral and parenteral insulin to prevent or delay type 1 diabetes.

\section{Procedures}

Each of the nine US DPT-1 centers obtained institutional review board approval to administer the surveys to participants $\geq 10 \mathrm{yr}$ of age and to parents of participants $<18 \mathrm{yr}$ of age at the beginning of the trial. Survey completion was voluntary and confidential. For children 10-17 yr old, the child's parent decided whether the survey should be given to the child. Every effort was made to administer the survey at the end of the trial before the results were publicized.

\section{Study sample}

Because knowledge of the trial results could bias response, only surveys of participants who reported no knowledge of trial results were analyzed: 124 participants, representing $43 \%$ of 
all participants $\geq 10 \mathrm{yr}$ of age at trial's end, and 219 parents of participants (131 mothers and 87 fathers) (1 parent did not self-identify gender), representing $48 \%$ of participants who began the trial as children. As expected, survey participants were older $(M=19.6 \pm 10.7)$ than the full trial participants $(M=15.9 \pm 8.9)$ because surveys were given only to those $\geq 10$ yr of age. Within the participant sample, $38 \%$ of the survey respondents were female, and within the parent sample, $34 \%$ of the parents' children were female, comparable to the $40 \%$ female participation rate in the full trial.

\section{Data analysis}

Survey item frequency distributions were reviewed and where appropriate, item responses were recoded to normalize the data. Highly correlated items were grouped into multiple item scales. $T$-tests, repeated measures analysis of variance, and multiple/logistic regression techniques were used to test study protocol (study procedure) and respondent (participant age, participant vs. parent, and gender) effects.

\section{Results}

\section{Distress at the beginning of the trial}

Most participants (81\%) and most parents (96\%) reported being upset with the news of their own or their child's increased diabetes risk. On a 0 - to 3 -point scale ( $0=$ not upset at all to 3 $=$ very upset $)$, parents $(\mathrm{M}=1.95 \pm 0.85)$ were more upset than participants $(\mathrm{M}=1.63 \pm$ $\left.1.09 ; \mathrm{t}_{340}=2.98, \mathrm{p}<0.01\right)$ and mothers $(\mathrm{M}=2.05 \pm 0.84)$ were more upset than fathers ( $\mathrm{M}$ $\left.=1.80 \pm 0.83 ; \mathrm{t}_{216}=2.14, \mathrm{p}<0.05\right)$. Child participants $(<18 \mathrm{yr}$ of age $)(\mathrm{M}=1.87 \pm 1.04)$ were more upset than adult participants ( $\geq 18 \mathrm{yr}$ of age $)\left(\mathrm{M}=1.27 \pm 1.07 ; \mathrm{t}_{121}=3.06, \mathrm{p}<\right.$ $0.01)$. Approximately half of participants (53\%) and parents (50\%) acknowledged being somewhat scared when they started the study. On a 5-point scale $(1=$ very happy to $5=$ very scared), child participants reported feeling more scared $(\mathrm{M}=3.69 \pm 0.83)$ than adult participants $\left(\mathrm{M}=3.30 \pm 0.79 ; \mathrm{t}_{108}=2.44, \mathrm{p}<0.05\right)$.

\section{Decision to participate in the trial}

Overall, respondents described the decision to participate in the trial as relatively easy. On a 5 -point scale $(1=$ very easy to $5=$ very hard $)$, participants $\left(\mathrm{M}=1.79 \pm 0.86 ; \mathrm{t}_{358}=3.58, \mathrm{p}<\right.$ $0.001)$ described the trial participation decision as easier than parents $(\mathrm{M}=2.18 \pm 0.97)$ and adult participants $\left(\mathrm{M}=1.50 \pm 0.75 ; \mathrm{t}_{111}=3.05, \mathrm{p}<0.001\right)$ reported the decision as easier than child participants $(\mathrm{M}=1.99 \pm 0.88)$.

\section{Beliefs about group assignment}

Participants were randomly assigned to oral insulin capsules or to a placebo. Because participants often have opinions about which condition they have been assigned, the survey asked whether the respondent could tell which type of capsule the participant was given. Participants were evenly divided between 'don't know' (55\%) and stating that they believed that they were in the placebo $(27 \%)$ or oral insulin (17\%) condition. In contrast, most parents (72\%) said that they did not know if the child was given the active drug, $18 \%$ believed that their child was given a placebo, and only $11 \%$ thought that the child was randomized to oral insulin. Participants were more likely to have an opinion about what 
condition they were in than parents $\left(\chi^{2}{ }_{1,343}=8.75, \mathrm{p}<0.01\right)$ and were more likely to believe that they were in the control group than parents $\left(\chi^{2}{ }_{1,343}=4.14, \mathrm{p}<0.05\right)$. Very few participants or parents believed that they were (or their child was) assigned to the oral insulin condition.

At the end of the survey, respondents were given a hypothetical question in which they could choose which of all the DPT-1 conditions (insulin injections, capsules, or no intervention) they would have preferred. Most participants (53\%) and parents (60\%) chose the capsule condition. More participants (21\%) than parents (8\%) chose the no intervention condition $\left(\chi^{2} 1,343=11.51, \mathrm{p}<0.001\right)$. Very few participants and parents $(3 \%)$ chose the insulin injection condition.

\section{Reactions to study procedures}

Each respondent answered three questions about each study procedure - how much it hurt (finger sticks and OGTT only, not capsules), how difficult it was, and how much they disliked it. Answers to the questions were highly correlated for a given procedure ( $a=0.58$ for capsules, 0.71 for the OGTT, and 0.64 for finger sticks for home blood glucose testing) and were combined into a single measure of procedure distress $(0=$ no distress, $1=$ some distress, and $2=$ great distress).

Each participant or parent was also asked whether he or she would be willing to participate in a future study with the same procedures $(0=$ no, $1=$ maybe, and $2=y e s)$ and to select the worst and best aspect of the study.

Study procedure distress-All participants experienced three study procedures: daily ingestion of capsules, finger sticks for blood glucose tests every 3 months, and an OGTT every 6 months. There was a significant interaction between respondent (parent/ participant) and study procedure $\left[F_{2,67}=16.85, \mathrm{p}<0.001\right]$. Parents rated the OGTT $(\mathrm{M}=0.98 \pm 0.50)$ as most distressing, followed by finger sticks $(\mathrm{M}=0.81 \pm 0.39)$; taking capsules $(\mathrm{M}=0.77$ $\pm 0.44)$ was rated as least distressing. In contrast, participants rated taking capsules $(\mathrm{M}=$ $0.92 \pm 0.48)$ as the most distressing study procedure, followed by finger sticks $(\mathrm{M}=0.80 \pm$ $0.45)$; the OGTT $(\mathrm{M}=0.76 \pm 0.50)$ was rated as the least distressing.

\section{Willingness to participate in future studies with similar procedures-}

Willingness to participate in another study with the same procedure provides a good overall indicator of respondent reaction to each study procedure, including random assignment. Figure 1 depicts the percentage of participants and parents answering 'yes' by study procedure. For comparison purposes, the same data from the parenteral arm of the DPT-1 (2) are provided. Both participants and parents were most willing to be in another study involving finger sticks (63\%) and least willing to participate in another study involving random assignment $(47 \%)\left(F_{3,975}=19.11, \mathrm{p}<0.001\right)$. Figure 2 provides the same data for child vs. adult participants. Again, for comparison purposes, the same data from the parenteral arm of the trial (2) are provided. Adult participants were significantly more willing to participate in finger sticks (73\%) and random assignment $(65 \%)$ than child participants (58\% finger sticks, $38 \%$ random assignment; $F_{3,339}=2.59$, $\mathrm{p}<0.05$ ). 
The worst and best part of the study-Asking respondents to select the single worst and best part of the study was another method used to identify procedures that were perceived to be particularly difficult or beneficial. For participants, the OGTT was selected as the worst part of the study (31\%), followed by worrying about getting diabetes (23\%). Child participants (32\%) were more likely than adult participants (8\%) to endorse worrying about getting diabetes $\left(\chi_{1,124}^{2}=9.09, \mathrm{p}<0.01\right)$. Parents most often endorsed worrying about their child getting diabetes (53\%) as the worst part of the study, followed by the OGTT (29\%). Parents endorsed worrying about diabetes significantly more often than participants $\left(\chi_{1,343}^{2}=4.14, p<0.05\right)$. Finger sticks or taking capsules were very rarely selected as the worst part of the study by participants or parents.

Most participants (57\%) and parents (93\%) stated that the best part of the study was knowing that someone was watching the participant for developing diabetes.

\section{Concerns about hypoglycemia}

Few respondents reported worrying that the study capsules would cause hypoglycemia. However, more parents (31\%) acknowledged worrying about hypoglycemia than participants $(18 \%)\left(\mathrm{t}_{329}=2.57, \mathrm{p}<0.01\right)$.

\section{Adherence with study procedures}

Blood glucose testing-All study participants were asked to do finger sticks every 3 months or when they suspected hypoglycemia. Blood glucose testing frequency reports were converted to a 4 -point scale ( $0=$ testing every 6 months or less, $1=$ testing every 3 months, $2=$ testing monthly or weekly, and $3=$ testing every day). Participants reported no significant change in blood glucose testing frequency over the course of the study (beginning: $\mathrm{M}=1.12 \pm 0.51$, end: $\mathrm{M}=1.18 \pm 0.74$ ); most participants (78\% at study beginning and $67 \%$ at study end) reported testing every 3 months, consistent with the study protocol. In contrast, parents reported a significant increase in testing frequency from the beginning $(\mathrm{M}=1.12 \pm 0.61)$ to the end of the study $\left(\mathrm{M}=1.34 \pm 0.91 ; \mathrm{t}_{176}=2.91, \mathrm{p}<0.01\right)$. At the beginning of the study, $72 \%$ of parents reported blood glucose testing every 3 months, $11 \%$ reported less frequent testing, and $16 \%$ reported more frequent testing. By the end of the study, $54 \%$ of parents reported testing every 3 months per study protocol but $34 \%$ were testing more frequently.

Capsule ingestion-Participants were instructed to take one capsule each day before breakfast. Nearly all participants reported taking their capsules in the morning (94\%), followed by evening (11\%) or afternoon (4\%). Most participants swallowed capsules by mouth $(91 \%)$, a few of them in drinks or food $(10 \%)$. Respondents were asked how often they missed capsules in a normal week at the beginning and end of the study. The mean number of capsules missed per week by participant report was $1.56 \pm 1.06$ at the study beginning and $4.34 \pm 2.79$ at the study end, a statistically significant increase $\left(\mathrm{t}_{100}=9.95, \mathrm{p}\right.$ $<0.001)$. The mean number of capsules missed per week by parent report also increased over the course of the study (1.24 \pm 0.59 capsules missed at study beginning and $1.89 \pm 1.44$ missed at study end; $\left.\mathrm{t}_{146}=5.21, \mathrm{p}<0.001\right)$. Participants reported more missed capsules than parents at both the beginning of the study $\left(\mathrm{t}_{287}=2.94, \mathrm{p}<0.001\right)$ and the end of the study 
$\left(\mathrm{t}_{268}=11.07, \mathrm{p}<0.001\right)$. At study end, $13 \%$ of participants and $3 \%$ of parents stated that the participant was not taking any capsules at all. In the 45 cases where both a mother and a child completed the survey, there was poor agreement between parent and child on the number of missed capsules ( $\mathrm{r}=0.16$ at study beginning and 0.30 at study end).

\section{Efforts to prevent or delay diabetes onset}

More participants (48\%) than parents (38\%) reported doing something to delay or prevent diabetes onset $\left(\chi^{2} 2,343=6.08, \mathrm{p}<0.05\right)$. Changing diet was the most common behavior reported (44\% of participants, $27 \%$ of parents), followed by increasing exercise (33\% of participants, $16 \%$ of parents) and taking alternative medicines, predominantly vitamins (23\% of participants, $10 \%$ of parents). More participants who thought that they were in the placebo condition $(54 \%)$ report engaging in a behavior change compared with other study participants $(40 \%)\left(\chi^{2} 1,343=4.16, \mathrm{p}<0.05\right)$.

\section{Need for psychological support}

Two survey items examined whether participants would have liked the opportunity to see a counselor or share their experiences with other study participants. Both participants and parents expressed a greater interest in sharing experiences with other DPT-1 participants ( $24 \%$ yes and $32 \%$ maybe) than seeing a counselor ( $8 \%$ yes and $19 \%$ maybe; $F_{1,342}=79.81$, $\mathrm{p}<0.001)$. More parents (9\% yes and $21 \%$ maybe) were interested in speaking to a counselor than participants $(5 \%$ yes and $15 \%$ maybe; Wald $=3.66, \mathrm{p}<0.05)$, and more female participants/parents ( $8 \%$ yes and $24 \%$ maybe) were interested in speaking to a counselor than male participants/parents $(7 \%$ yes and $14 \%$ maybe; Wald $=5.23, \mathrm{p}<0.05$ ).

\section{Overall reaction to study participation}

Three survey items examined overall reactions to study participation: 'Overall, how do you feel about being in the DPT-1?' ( $0=$ liked it a lot, $1=$ neutral, and $2=$ disliked it a lot $)$; 'Do you think being in the DPT-1 was a good decision?' $(0=$ a great decision, $1=$ neutral, and 2 = a bad decision $)$; and 'Would you recommend it to a friend?' $(0=$ yes, $1=$ maybe, and $2=$ no). The items were highly correlated $(\alpha=0.72)$ and were combined into a single score. Respondents expressed generally positive views toward the trial. Only $8 \%$ of participants and $6 \%$ of parents stated that they disliked the trial, $1 \%$ of participants and $2 \%$ of parents thought that it was a bad decision to participate, and $7 \%$ of participants and $4 \%$ of parents stated that they would not recommend it to a friend. However, mothers $(\mathrm{M}=0.26 \pm 0.39)$ were more favorable toward the study than fathers $(M=0.39 \pm 0.47), \mathrm{t}_{161}=-2.12, \mathrm{p}<$ $0.05)$.

\section{Beliefs about oral and parenteral insulin's effectiveness in preventing or delaying diabetes onset}

Two items assessed participant views about insulin injections and oral insulin as methods to delay or prevent diabetes onset. Both were rated on a 3-point scale $(0=$ no, $1=$ don't know or maybe, and $2=$ yes). Participants and parents who believed that the participant received the active drug were more likely to believe that oral insulin can delay diabetes onset $(\mathrm{M}=$ $1.18 \pm 0.62)$ or prevent it entirely $(\mathrm{M}=1.14 \pm 0.59)$ compared with all other respondents $(\mathrm{M}$ 
$=0.94 \pm 0.48$ for delay and $\mathrm{M}=0.77 \pm 0.51$ for prevent; $\mathrm{t}_{340}=2.94, \mathrm{p}<0.01$ for delay, and $\mathrm{t}_{340}=4.35, \mathrm{p}<0.001$ for prevent). Participants were more likely to believe that oral insulin could delay $(\mathrm{M}=1.11 \pm 0.56)$ or prevent diabetes $(\mathrm{M}=0.94 \pm 0.53)$ compared with parents $\left(\mathrm{M}=0.90 \pm 0.45\right.$ for delay and $\mathrm{M}=0.75 \pm 0.52$ for prevent; $\mathrm{t}_{340}=3.87, \mathrm{p}<0.001$ for delay, and $t_{340}=3.28, p=0.001$ for prevent). Child participants were more likely to believe that oral insulin could prevent diabetes entirely $(\mathrm{M}=1.17 \pm 0.55)$ compared with adult participants $(\mathrm{M}=1.02 \pm 0.56 ; \mathrm{t} 121=2.21, \mathrm{p}<0.05)$.

When asked about whether parenteral insulin can delay or prevent diabetes, respondents were less likely to believe in its effectiveness compared with oral insulin (parenteral insulin $\mathrm{M}=0.82 \pm 0.57 \mathrm{vs}$. oral insulin $\mathrm{M}=0.98 \pm 0.50$ for delaying diabetes; $F_{1,339}=31.44, \mathrm{p}<$ 0.001 , and parenteral insulin $\mathrm{M}=0.61 \pm 0.56$ vs. oral insulin $\mathrm{M}=0.82 \pm 0.53$ for preventing diabetes; $\left.F_{1,336}=33.61, \mathrm{p}<0.001\right)$.

\section{Discussion}

Findings from this study are consistent with our prior report of participant and parent experiences in DPT-1's parenteral prevention trail (2). Participant characteristics - age, parental role, and gender - were all associated with important differences in trial experiences. Compared with participants, parents in both DPT-1 prevention trials were more distressed at the news of the child's increased diabetes risk, worried more about hypoglycemia during the trial, were more likely to report worrying about the child's getting diabetes as the worst aspect of the study, and were more likely to express an interest in meeting with a counselor during the trial. Mothers in both DPT-1 prevention trials, compared with fathers, reported greater distress, and females reported a greater interest in meeting with a counselor than males. These findings indicate that emotional distress associated with a prevention trial may vary by gender and parental role, suggesting that trial investigators should give increased consideration to the emotional needs of these particular study participants.

Certain study procedures were common to both the DPT-1 prevention trials, and the consistency of participants' views toward these procedures is striking. In both the oral and the parenteral trials, finger sticks was the procedure participants viewed as most acceptable (Fig. 1). In contrast, more than half of participants and parents stated that they would not join another study requiring random assignment (Fig. 1). Only adults in the oral trial viewed this component of the study design favorably (Fig. 2). These findings suggest that random assignment - the hallmark of clinical trial design - may be a serious impediment to study recruitment. Increased attention needs to be given to educating the public, as well as trial participants, about the purpose and importance of randomizing participants to study conditions.

Figure 2 also highlights the consistent differences between children and adults in their attitudes toward study procedures. For every procedure, adult participants were more likely to agree to another trial with that procedure than child participants. However, the differences were greatest in the parenteral prevention trial. The DTP-1 parenteral study involved more invasive procedures including insulin injections and 4-d insulin infusions, which may have 
highlighted child vs. adult participant differences. For every procedure except random assignment, the majority of adult and child participants in the oral trial stated that they would agree to be in another trial with that procedure. This was never the case for children in the parenteral trial. These findings suggest that while most adults may be amenable to both invasive (e.g., insulin injections) and non-invasive (oral medications) clinical trials, the concerns of children require greater consideration.

Examination of study adherence reported by participants in both DPT-1 prevention trials highlights a number of commonalities. Parents in both trials reported a significant increase in blood glucose testing over the course of the study. Parents worry more about possible diabetes onset in the child and may conduct blood glucose tests as a way of monitoring the child for developing diabetes. Previous reports suggest that parental blood glucose monitoring of unaffected siblings in families with a diabetic child is relatively common (4, $5)$.

Declining adherence with the study medication was a problem in both the DPT-1 prevention trials, and participants consistently reported significantly more missed medication than parents. At the end of the oral trial, participants reported an average of 4.3 capsules missed per week or $61 \%$ of the prescribed dose; parents reported an average of 1.9 capsules missed per week or $27 \%$ of the prescribed dose. In the parenteral trial, participants reported an average of 3.5 injections missed per week at study end, but because the protocol required two injections per day, this amounted to $25 \%$ of the prescribed dose. Parents in the parenteral trial reported 2.3 injections missed per week at study end or $16 \%$ of the prescribed dose. Many participants in both the parenteral (15\%) and the oral (13\%) DPT-1 trials reported taking no insulin at all at the end of the study. While parents seemed to be aware of missed injections in the parenteral trial, they underestimated missed capsules in the oral insulin study. Parents and children exhibited excellent agreement on the number of injections missed in the parenteral trial but poor agreement in the oral study. It is likely that parents more closely supervised children in the parenteral trial because insulin injections were more invasive and more often required parental assistance; they were also strongly disliked by child participants. In contrast, children in the oral trial may have been expected to take the capsules on their own, with parental supervision declining over time. While parents rated taking capsules as the least distressing procedure in the oral trial, participants rated it as the most distressing. Although children in the oral trial clearly rated taking capsules as more acceptable than children in the parenteral trial rated taking insulin injections (Fig. 2), many children disliked taking capsules and appeared to have skipped doses without their parents' knowledge. Long-term adherence with a study protocol is clearly a challenge, and one that needs greater attention in terms of both mechanisms to promote continued adherence and ways to include such data in the interpretation of study findings.

Confirming previous reports $(4,6)$, participants and parents in both DPT-1 prevention trials acknowledged engaging in a variety of behaviors to delay or prevent diabetes onset, with dietary and activity changes most commonly reported. Efforts to stop or delay the disease were more often acknowledged among parenteral trial participants (57\%) and parents (48\%) compared with oral trial participants (48\%) and parents (38\%). This may be a function of the 
higher diabetes risk that characterized the parenteral sample. Those as higher risk may be more motivated to try one or more interventions outside the study protocol in an effort to delay or prevent the disease. In the oral trial, participants who believed that they were randomized to the placebo arm were particularly likely to report efforts to prevent or delay the disease. In the parenteral arm, significantly more respondents assigned to the closely monitored group reported using alternative medicines compared with those assigned to the intervention group. These findings suggest that participants' beliefs about the power of the study condition to which they are assigned may influence willingness to engage in behaviors outside the study protocol to delay or prevent the disease. The fact that large numbers of participants engage in behaviors outside a clinical trial presents a threat to the internal validity of any trial and complicates interpretation of results. At a minimum, the extent and nature of these participant-initiated behaviors should be monitored.

In both DPT-1 prevention trials, actual study assignment or beliefs about study assignment influenced respondents' confidence in a specific intervention's potential to prevent or delay type 1 diabetes. In the parenteral trial, those randomized to the intervention condition were more confident that insulin could stop diabetes compared with those assigned to the closely monitored group. Although all respondents in the oral trial had greater confidence in oral insulin than parenteral insulin as a mechanism of disease prevention, those who believed that they were randomized to the active drug condition were particularly favorable. Parental role and participant age were again important considerations in respondents' views toward the study intervention. In both prevention trials, children were more confident that the study intervention would succeed compared with adults. Parent expectations were quite different between the two trials. In the parenteral trial, parents were more confident than participants in the intervention's potential for success. The opposite finding emerged in the oral trial, where participants were more favorable. It is important to note that participants knew their study condition in the parenteral trial, while the oral trial involved blind random assignment. Fewer parents in the oral trial believed that their child was in the oral insulin condition, compared with participants, which may explain parents' lower confidence in oral insulin's potential for success.

Only in the oral prevention trial survey, did we provide participants with a hypothetical question in which they could choose which of the DTP-1 conditions (insulin injections, oral capsules, or no intervention) they would have preferred. It is not surprising that most participants and parents chose the oral medication condition. It appears that most participants develop positive attitudes toward the study condition to which they have been assigned.

Participants and parents in both DPT-1 prevention trials reported that knowing that someone was watching the participant for developing diabetes was the best part of the study. Participants' overall reactions to the study were very favorable in both DPT-1 prevention trials, highlighting the positive perceptions most DPT-1 participants had of their study experience.

Study results should be interpreted with some caution in view of the special characteristics of the sample surveyed. Only participants $\geq 10 \mathrm{yr}$ participated, so the study provides no 
information on the experiences of younger children except from their parents' point of view. All participants were relatives of patients with type 1 diabetes, limiting our ability to generalize our findings to the general population. Those surveyed are likely to be the most dedicated to the study and certainly are not representative of those who refused the trial entirely. Recalled reactions to study components may differ from participants' or parents' immediate reactions during the study.

Despite these limitations, the survey results reported in this study and previously (2) are clearly relevant to the oral insulin trial being initiated as part of TrialNet and to other diabetes prevention studies involving children. Specific recommendations for those designing future prevention trials include (i) increase public and trial participant education on the value of random assignment; (ii) provide mechanisms to address participant and parent emotional distress when it occurs; (iii) address the particular concerns of children in the selection of study procedures; (iv) monitor protocol adherence, include adherence promotion strategies as part of the study protocol, and include adherence data in the study's data analysis and interpretation of the study's findings; (v) monitor participant behaviors initiated outside the study protocol aimed at delaying or preventing disease onset, consider strategies to reduce this type of behavior, and include these data in the study's data analysis and interpretation of the study's findings.

\section{Acknowledgments}

The DPT-1 was supported by the National Institutes of Health, National Center for Research Resources, American Diabetes Association, and Juvenile Diabetes Research Foundation.

\section{References}

1. Diabetes Prevention Trial - Type 1 Diabetes Study Group. Effects of insulin in relatives of patients with type 1 diabetes mellitus. N Engl J Med. 2002; 346:1685-1691. [PubMed: 12037147]

2. Johnson SB, Baughcum AE, Hood K, Rafkin-Mervis LE, Schatz DA. for the DPT-1 Study Group. Participant and parent experiences in the parenteral insulin arm of the Diabetes Prevention Trial for Type 1 Diabetes. Diabetes Care. 2007; 30:2193-2198. [PubMed: 17563348]

3. Diabetes Prevention Trial - Type 1 Diabetes Study Group. Effects of oral insulin in relatives of patients with type 1 diabetes mellitus. Diabetes Care. 2005; 28:1068-1076. [PubMed: 15855569]

4. Baughcum A, Johnson SB, Carmichael S, Lewin A, She J-X, Schatz D. Maternal efforts to prevent type 1 diabetes in at-risk children. Diabetes Care. 2005; 28:916-921. [PubMed: 15793195]

5. Lucidarme N, Domingues-Muriel E, Castro D, Czernichow P, Levy-Marshall C. Appraisal and implications of predictive testing for insulin-dependent diabetes mellitus. Diabetes Metab. 1998; 23:550-553. [PubMed: 9932224]

6. Johnson SB, Tercyak K. Psychological impact of islet cell antibody screening for IDDM on children, adults, and their family members. Diabetes Care. 1995; 18:1370-1372. [PubMed: 8721939] 


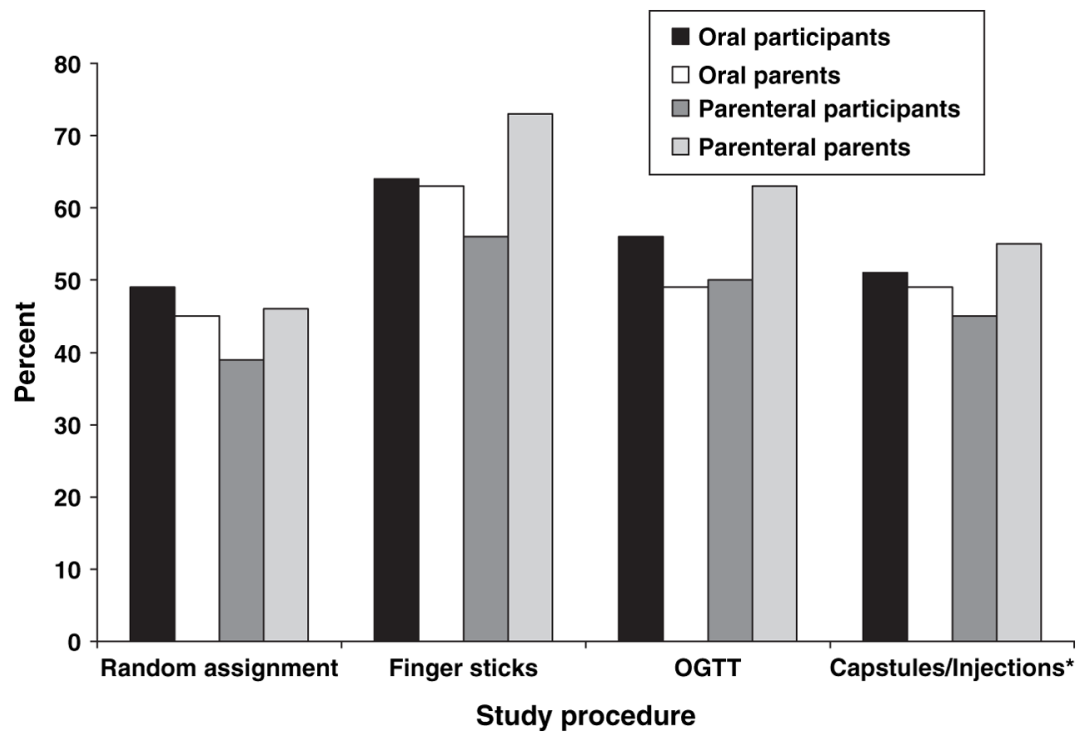

Fig. 1.

Percent of oral and parenteral trial participants and parents willing to be in another study by study procedure. *In the oral trial, medication was given by capsule; in the parenteral trial, medication was given by injection. OGTT, oral glucose tolerance test. 


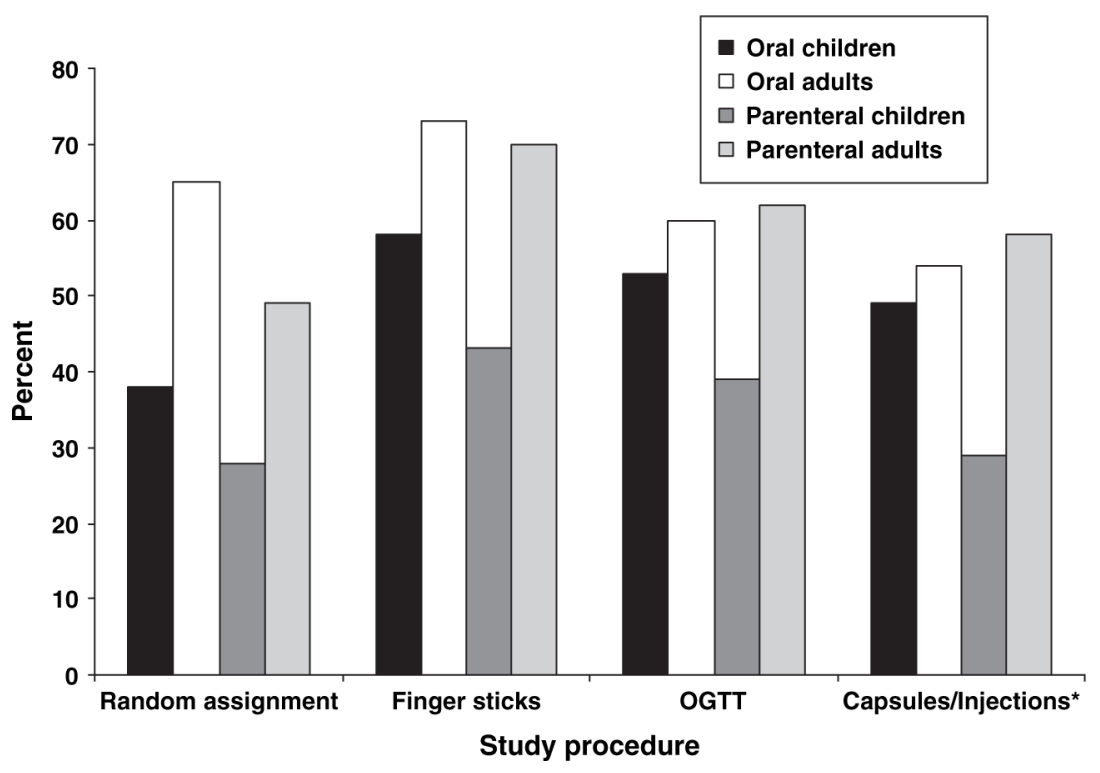

Fig. 2.

Percent of oral and parenteral trial child and adult participants willing to be in another study by study procedure. *In the oral trial, medication was given by capsule; in the parenteral trial, medication was given by injection. OGTT, oral glucose tolerance test. 